\title{
Enhancing learning of ENT examinations using online videos
}

\author{
J Buckley, S Carrie. Freeman Hospital
}

\section{Background}

In common with other medical schools, students at Newcastle University have limited practical ENT exposure during their undergraduate training. Using digital media to aid learning has demonstrated improved knowledge gains in medicine ${ }^{1}$. Training videos could provide a foundation of learning and a useful revision aid, allowing students to optimise their learning from clinical exposure.

\section{Objective}

To create an online resource facilitating undergraduate learning of ENT examinations and assess their effectiveness.

\section{Method}

The Newcastle University undergraduate curriculum was reviewed to determine ENT examination learning objectives. The chosen examination skills were then planned and filmed using ENT team members as actors. Voice-overs were created detailing key points and important steps of each examination. The videos were peer reviewed within the ENT department.

A link to a survey monkey questionnaire was sent to all third year medical students at Newcastle University and the results analysed.

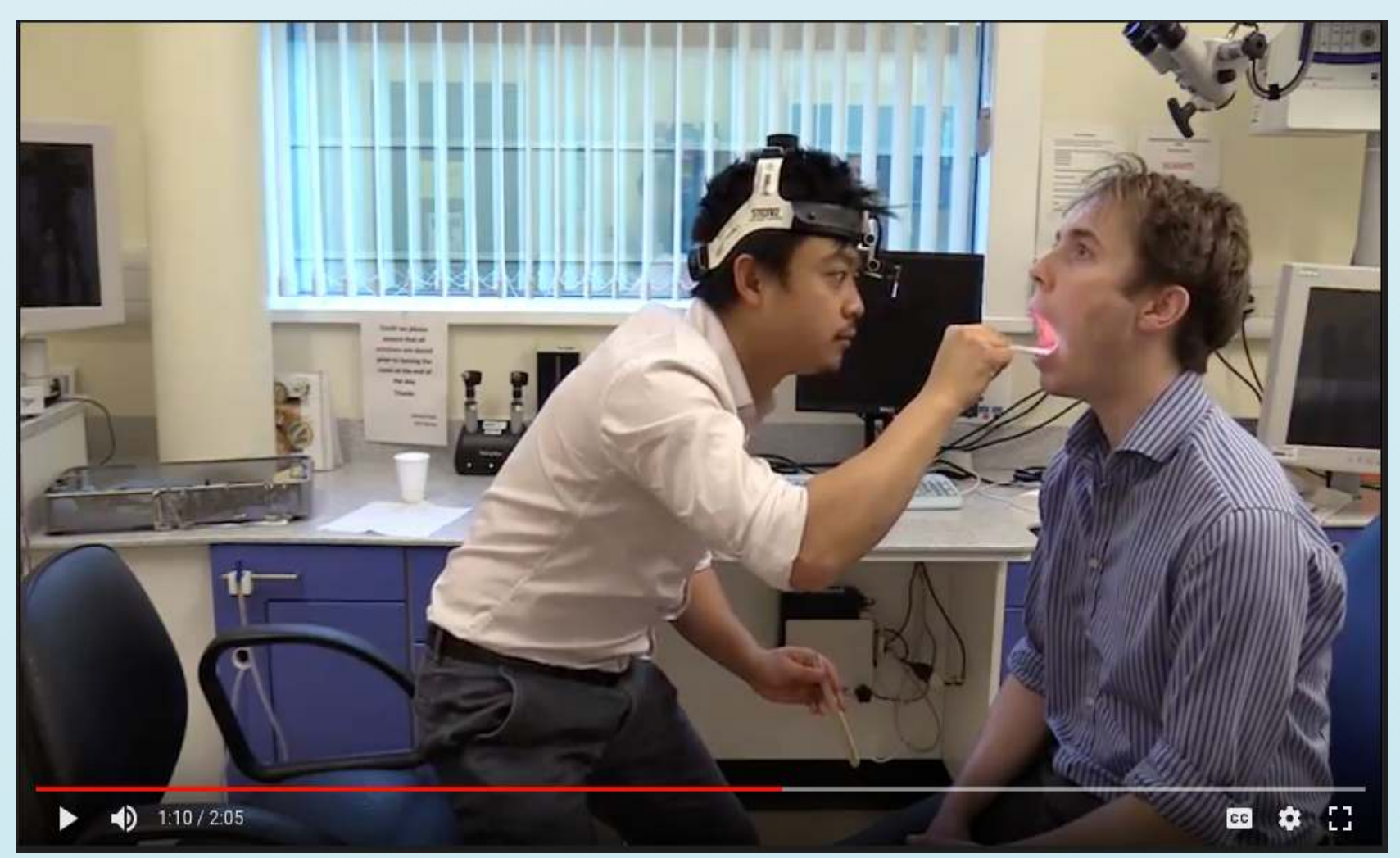

\section{Results}

The questionnaire was sent to 200 students, ten students completed the survey. The feedback was positive with those students reporting the videos improved their knowledge and confidence to perform ENT examinations. The students felt the videos were set an appropriate level and would make a useful revision resource. The response rate was low despite reminder emails.

\section{I feel more confident in my ability to perform ENT examinations}

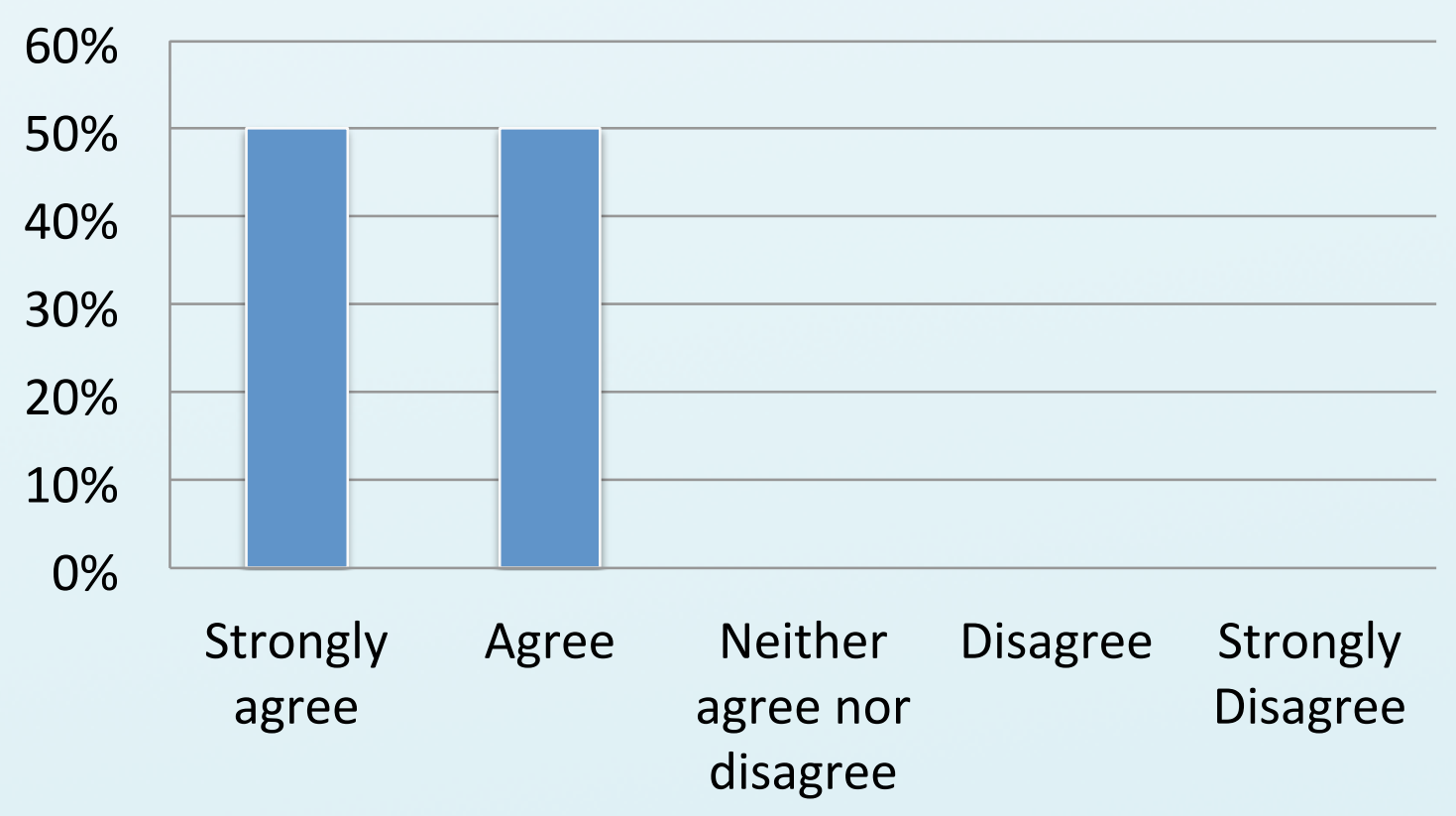

My knowledge of how to perform ENT examinations has improved

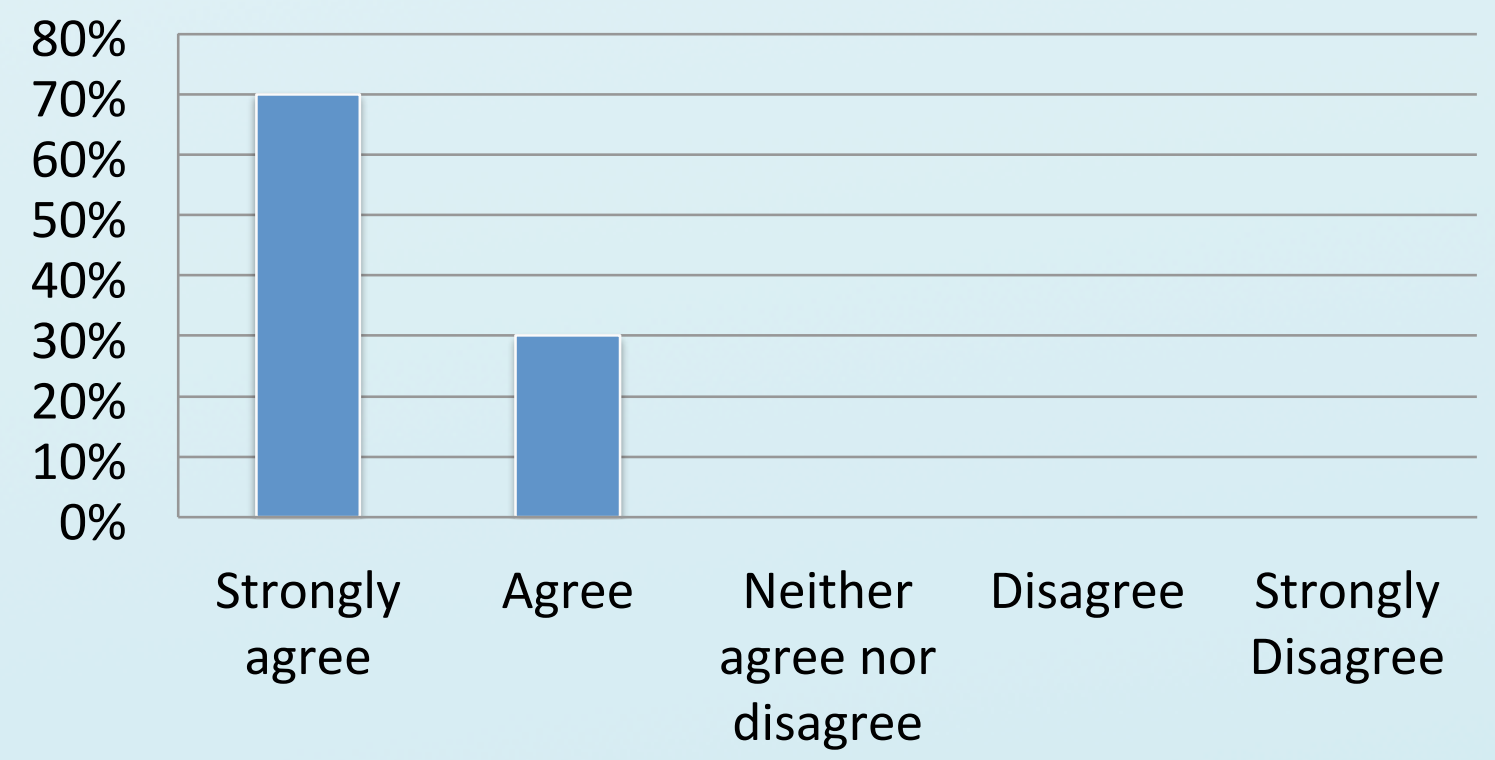

\section{Conclusion}

Medical students have found educational videos to have a positive impact on their confidence and knowledge of ENT examinations. There may be further scope to develop this resource using student feedback and peer review to guide development.

\section{References}

1. Superior gain in knowledge by podcast versus text-based ;learning in teaching orthopaedics: A Randomized controlled Trial. Black D, Von Malotky, Sostmann K, Hube R, Peters $\mathrm{H}$ and Hoff E. Journal of Surgical Education. 2016. 74, 1; 154-160 